Original Research Paper

\title{
Our Universe
}

\author{
${ }^{1}$ Relly Victoria Petrescu, ${ }^{2}$ Raffaella Aversa, ${ }^{3}$ Bilal Akash, ${ }^{4}$ Juan Corchado, \\ ${ }^{2}$ Antonio Apicella and ${ }^{1}$ Florian Ion Tiberiu Petrescu \\ ${ }^{1}$ ARoTMM-IFToMM, Bucharest Polytechnic University, Bucharest, (CE) Romania \\ ${ }^{2}$ Advanced Material Lab, Department of Architecture and Industrial Design, \\ Second University of Naples, 81031 Aversa (CE) Italy \\ ${ }^{3}$ Dean of School of Graduate Studies and Research, American University of Ras Al Khaimah, UAE \\ ${ }^{4}$ Union College, USA
}

Article history

Received: 03-06-2017

Revised: 05-06-2017

Accepted: 15-06-2017

Corresponding Author: Florian Ion Tiberiu Petrescu ARoTMM-IFToMM, Bucharest Polytechnic University, Bucharest, (CE), Romania E-mail: scipub02@gmail.com

\begin{abstract}
It's hard to know ourselves and our role as humanity, without knowing our precise location first. In the universe where we find ourselves (what we know not much about), there are billions of galaxies. A galaxy is a large cluster of stars (suns), i.e., solar systems; on average an ordinary galaxy contains about two billion stars (suns), which may or may not have planets around them. A constellation is a group of galaxies that depend on each other. Virgo is a very famous zodiacal constellation. Her name comes from Latin, the virgin and her symbol is $\mathrm{m}$. The constellation of the Virgin is located between the Lion to the west and the Libra to the east, being the second constellation in the sky (after Hydra) in size. The constellation of the Virgin can easily be observed in the sky of the earth due to its sparkling star named Spica. So our universe contains about two billion galaxies and many constellations; a constellation comprises several galaxies and a galaxy has about 2 billion stars. Nowadays, thanks to modern telescopes, we know a great many galaxies, but only 88 constellations have been identified by humanity (for now).
\end{abstract}

Keywords: Universe, Galaxy, Stars, Suns, Planets, Constellation, Virgin Constellation, Spica, Sombrero Galaxy, Milky Way, Sun Helios

\section{Introduction}

It's hard to know ourselves and our role as humanity, without knowing our precise location first.

In the universe where we find ourselves (what we know not much about), there are billions of galaxies.

A galaxy is a large cluster of stars (suns), ie solar systems; on average an ordinary galaxy contains about two billion stars (stars), which may or may not have planets around them Fig. 1.

Red is a skeleton positioned right in the heart of our universe. It is made up of several independent galaxies and constellations (a constellation is a group of galaxies that depend on each other); (Atkinson, 2015).

His name, skeleton, suggests that these galaxies are not scattered randomly in the universe we live in, but are put in a certain logical order, like a mammal skeleton.

The galaxy in which we find ourselves is called the "Milky Way", that is, the "Milk Way".

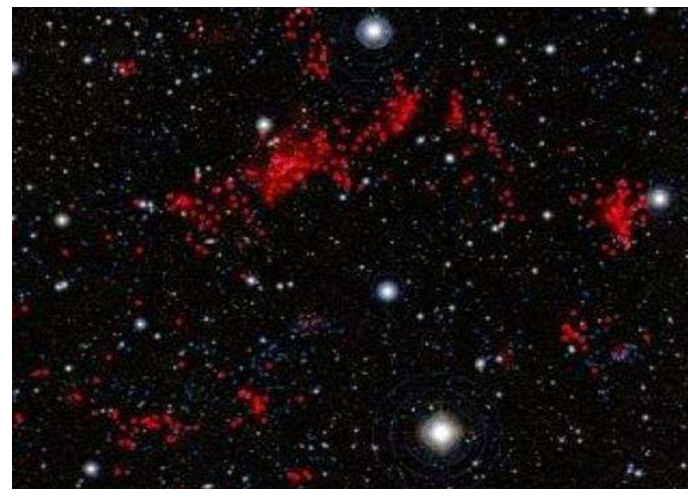

Fig. 1. Ou Universe. Source: https://www.universetoday.com/43990/do-skeletonfilaments-give-structure-to-the-universe/

Several dependent galaxies can form a constellation together. 
The constellation to which our galaxy belongs is called "The Constellation of the Virgin"; Fig. 2.

Virgo is a very famous zodiac constellation. Her name comes from Latin, the virgin and her symbol is mp (Virgo constellation From Wikipedia).

The constellation of the Virgin is located between the Lion to the west and the Libra to the east, being the second constellation in the sky (after Hydra) in size.

The constellation of the Virgin can easily be observed in the sky of the earth due to its sparkling star named Spica.

There are 35 verified exoplanets orbiting 29 stars in Virgo, including PSR B1257+12 (three planets), 70 Virginis (one planet), Chi Virginis (one planet), 61 Virginis (three planets), NY Virginis (two planets) and 59 Virginis (one planet).

Over 75 million light-years away in the constellation of Virgo (The Virgin) lies NGC 4981-a spiral galaxy with a rather explosive past.

NGC 4981 was discovered on 17 April 1784 by William Herschel and subsequently documented in John Dreyer's New General Catalogue. Over a century later, on 23 April 1968, the galaxy once again made it into the records when a Type la supernova-a stellar explosion in a binary star system-occurred within its confines: SN 1968I. SN 1968I, however, was not to be the galaxy's only supernova.

Decades later, the core collapse of a massive star led to supernova SN 2007c.

This spectacular shot of NGC 4981-not showing any of the supernovae explosions; the bright star visible in the image is a foreground star-was captured by FORS, the visible and near-UV FOcal Reducer and low dispersion Spectrograph for ESO's Very Large Telescope (VLT). FORS is the Swiss Army knife of ESO's instruments-it is able to study many different astronomical objects in many different ways and is responsible for some of the most iconic photos ever captured with the VLT (see eso9948f and eso0202a).

The data to create this image was selected from the ESO archive by Josh Barrington as part of the Hidden Treasures competition Fig. 3.

A noted galaxy that is not part of the cluster is the Sombrero Galaxy $\left(\mathrm{M}_{104}\right.$; Fig. 4), an unusual spiral galaxy. It is located about $10^{\circ}$ due west of Spica.

The famous Sombrero galaxy $\left(\mathrm{M}_{104}\right)$ is a bright nearby spiral galaxy. The prominent dust lane and halo of stars and globular clusters give this galaxy its name. Something very energetic is going on in the Sombrero's center, as much X-ray light has been detected from it. This X-ray emission coupled with unusually high central stellar velocities causes many astronomers to speculate that a black hole lies at the Sombrero's center-a black hole a billion times the mass of our Sun.

The nucleus of the Sombrero galaxy is classified as a Low Ionization Nuclear Emission Region (LINER). These are nuclear regions where ionized gas is present, but the ions are only weakly ionized (i.e., the atoms are missing relatively few electrons).

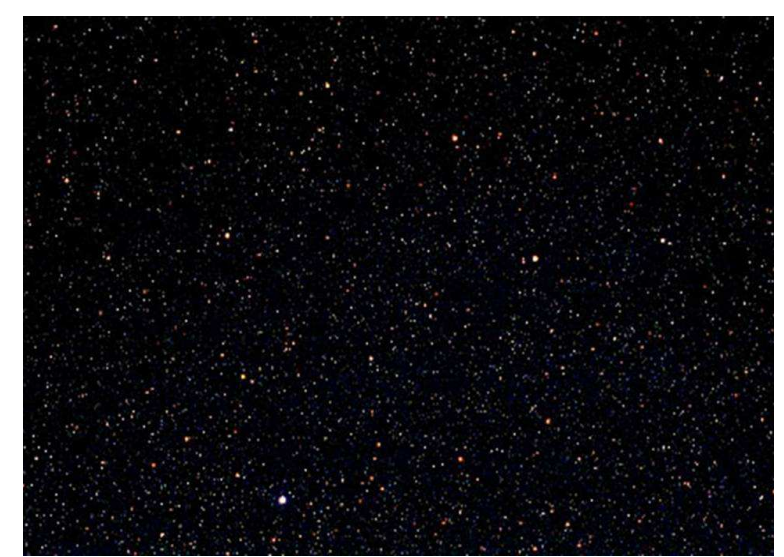

(a)

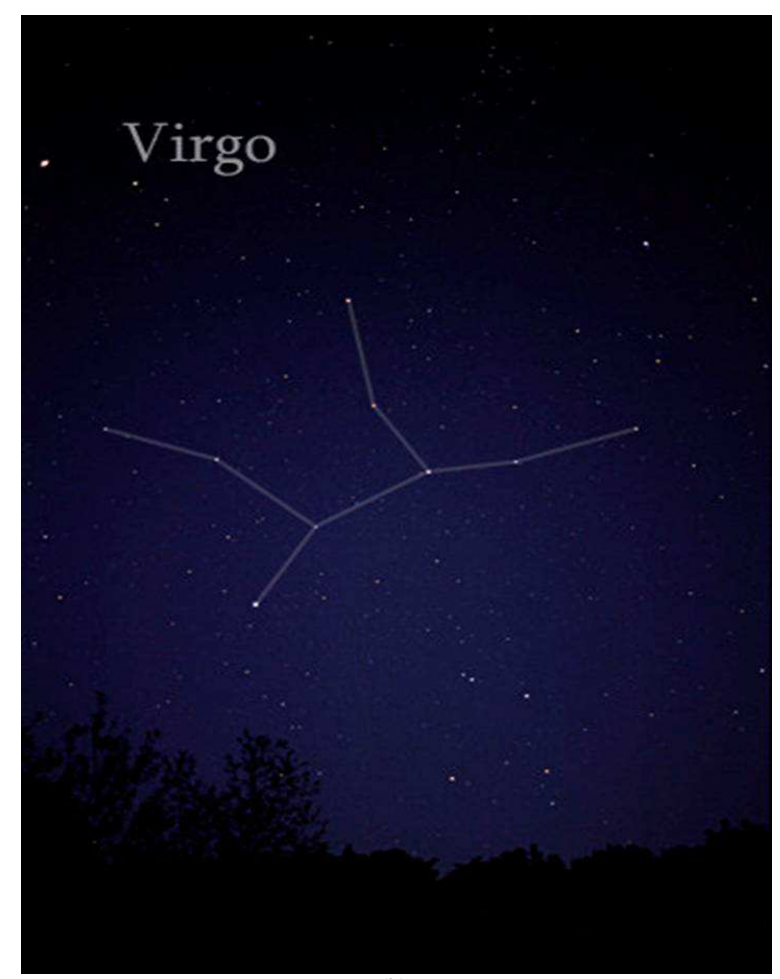

(b)

Fig. 2. The Virgo constellation (a) Virgo is a very famous zodiac constellation (b) Virgo constellation seen from the Earth. Source:

https://en.wikipedia.org/wiki/Virgo_(constellation)\#/medi a/File:VirgoCC.jpg

The source of energy for ionizing the gas in LINERs has been debated extensively. Some LINER nuclei may be powered by hot, young stars found in star formation regions, whereas other LINER nuclei may be powered by active galactic nuclei (highly energetic regions that contain supermassive black holes). Infrared spectroscopy observations have demonstrated that the nucleus of the Sombrero Galaxy is probably devoid of any significant star formation activity. 


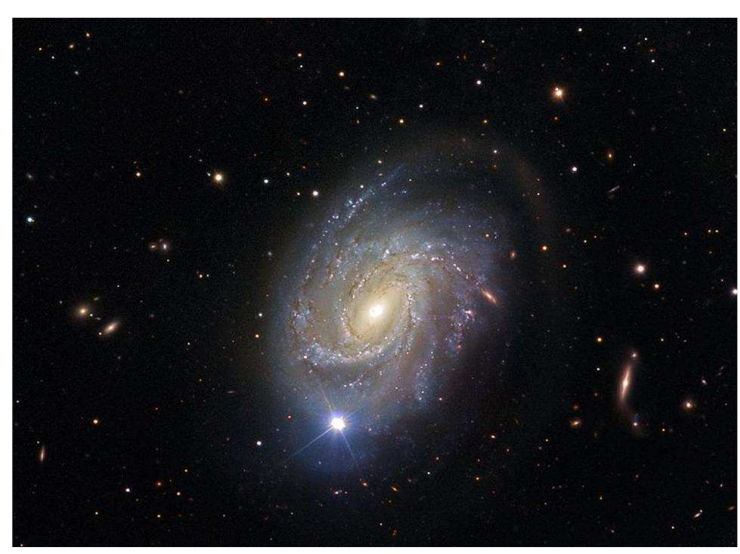

Fig. 3. NGC 4981 was discovered on 17 April 1784 by William Herschel.

Source: https://en.wikipedia.org/wiki/Virgo_(constellation)\#/me dia/File:When_Stars_Explode_NGC_4981.jpg

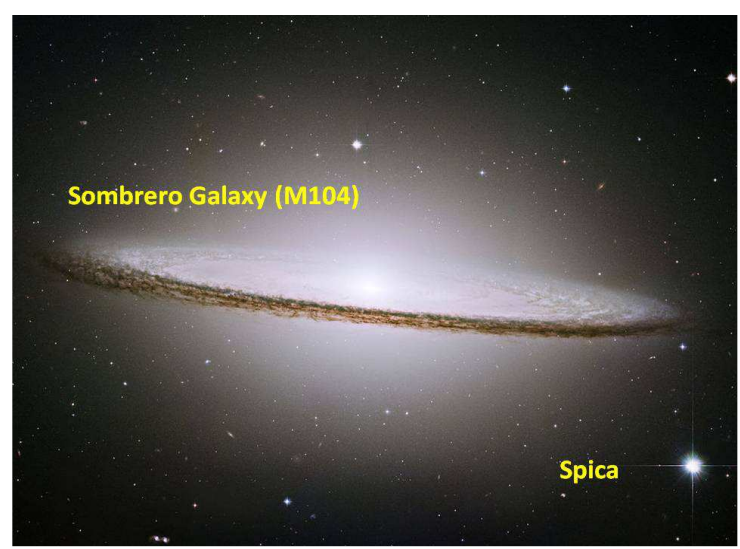

Fig. 4. Sombrero Galaxy $\left(\mathrm{M}_{104}\right)$, an unusual spiral galaxy, it is located about $10^{\circ}$ due west of Spica. Source: https://en.wikipedia.org/wiki/Sombrero_Galaxy\#/media /File:M104_ngc4594_sombrero_galaxy_hi-res.jpg

However, a supermassive black hole has been identified in the nucleus (as discussed in the subsection below), so this active galactic nucleus is probably the energy source that weakly ionizes the gas in the Sombrero Galaxy.

In the 1990s, a research group led by John Kormendy demonstrated that a supermassive black hole is present within the Sombrero Galaxy. Using spectroscopy data from both the CFHT and the Hubble Space Telescope, the group showed that the speed of revolution of the stars within the center of the galaxy could not be maintained unless a mass 1 billion times the mass of the Sun is present in the center. This is among the most massive black holes measured in any nearby galaxies.

Messier 84 (also known as M84 or NGC 4374) is an elliptical or lenticular galaxy in the constellation Virgo. In the Catalogue of Named Galaxies, it is called Ductrix Virginis, or the guide galaxy, after its position at the beginning of Markarian's Chain. M84 is situated in the heavily populated inner core of the Virgo Cluster of galaxies Fig. 5.

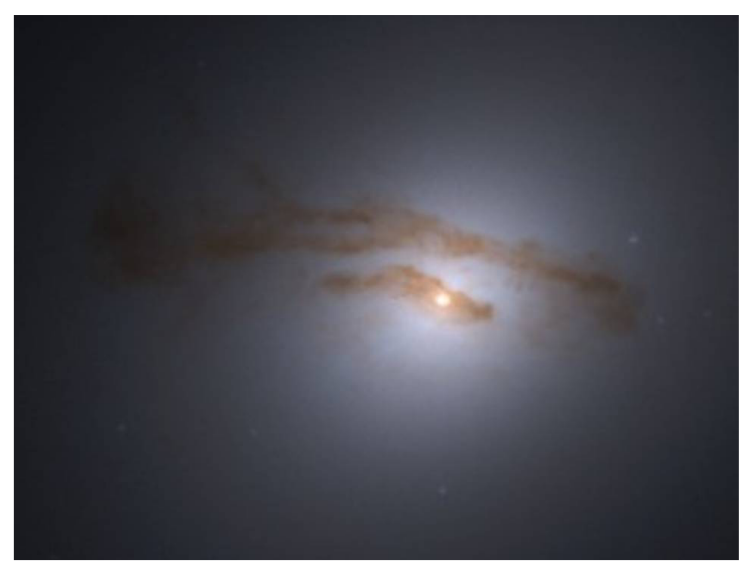

Fig. 5. Messier 84 (also known as M84 or NGC 4374) is an elliptical or lenticular galaxy in the constellation Virgo. Source:

https://en.wikipedia.org/wiki/Messier_84\#/media/File: Messier_84_nucleus_Hubble.jpg

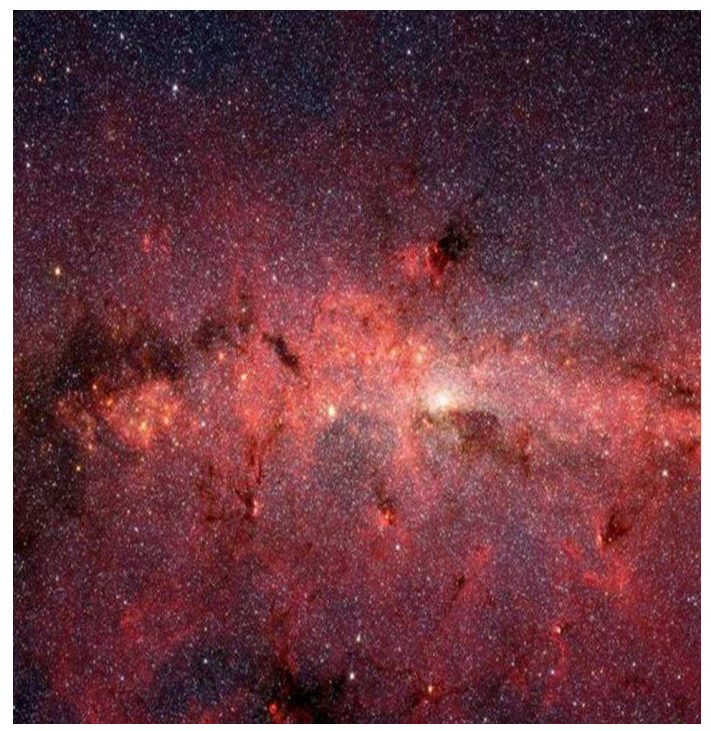

Fig. 6. The center (heart) of our galaxy has a bright and white look like milk. Source: https://en.wikipedia.org/wiki/Galactic_Center\#/media/F ile:Milky_Way_IR_Spitzer.jpg

Radio observations and Hubble Space Telescope images of M 84 have revealed two jets of matter shooting out from the galaxy's center as well as a disk of rapidly rotating gas and stars indicating the presence of a $1.5 \times 109 \mathrm{M} \odot$ supermassive black hole. It also has a few young stars and star clusters, indicating star formation at a very low rate.

Charles Messier discovered Messier 84 on 18 March 1781 in a systematic search for "nebulous objects" in the night sky. The object is the 84th in the Messier Catalogue (Charles Messier, from Wikipedia).

Two supernovae have been observed in M84: SN 1957 and SN 1991bg. Possibly, a third, SN 1980I is part of M84 or, alternatively, one of its neighboring galaxies, NGC 4387 and $\mathrm{M}$ 86. So our universe contains about two billion 
galaxies and many constellations; a constellation comprises several galaxies and a galaxy has about 2 billion stars. Nowadays, thanks to modern telescopes, we know a great many galaxies, but only 88 constellations have been identified by humanity (for now). Let's now return to our galaxy, where we are located and which is called the "Milky Way," the "Milk Way" Fig. 6.

\section{Methods and Materials}

In the Universe, in the "Constellation of the Virgin", in the "Milky Way Galaxy," there is also a sun (a star) called "Sun Helios" or simply "the Sun"; Fig. 7.

Helios is a true solar system made up of several planets that revolve around it.

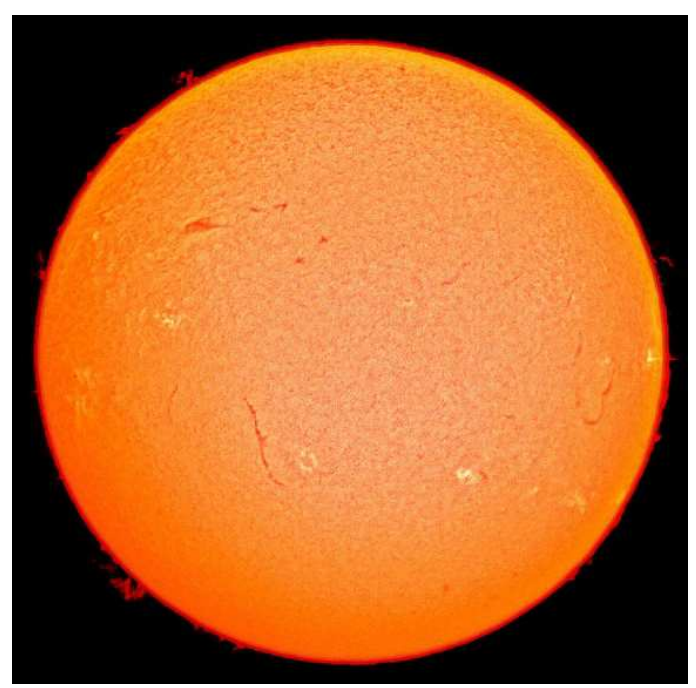

Fig. 7. Our "Sun, Helios" Source: https://en.wikipedia.org/wiki/Solar_System\#/media /File:Sun_in_February_(black_version).jpg

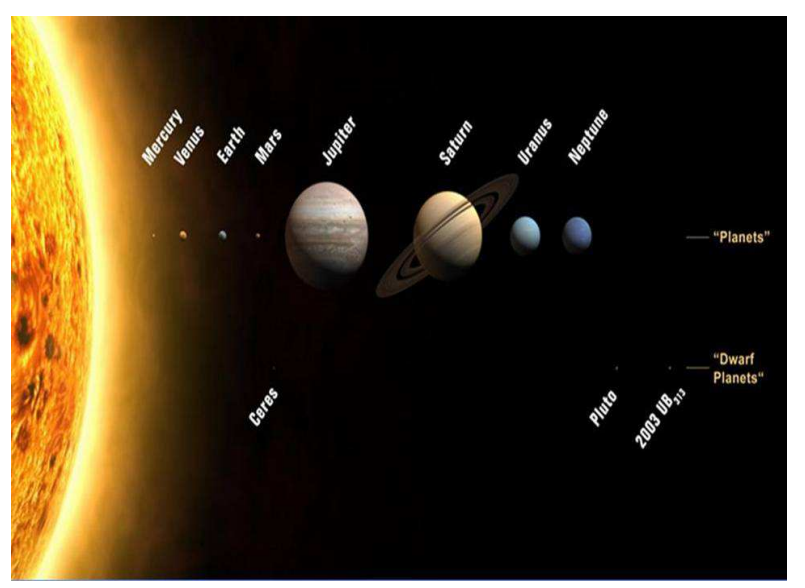

Fig. 8. Helios is a true solar system made up of several planets that revolve around it. The eight planets of our solar system are in order, starting from the Sun to the outside: Mercury, Venus, Earth, Mars, Jupiter, Saturn, Uranus and Neptune Source: https://en.wikipedia.org/wiki/Solar_System
The eight planets of our solar system Fig. 8 are in order, starting from the Sun to the outside: Mercury, Venus, Earth, Mars, Jupiter, Saturn, Uranus and Neptune. Some believe that Pluto is the new planet of our solar system, but it is as small as Ceres or UB313, which are considered to be asteroids, satellites, etc., that is, anything but planets (it is easy to assume that it was attracted By Helios and remained to gravitate around it like a planet, but it was initially a simple asteroid).

The first easy observation is that the main halo covers only the first two planets of Mercury and Venus, but it is much closer to the next two Earths and Mars.

There are more runes of some more dissipated halos batting Jupiter.

From Saturn to Neptune, the solar radiation of light and heat is greatly diminished.

Even from Jupiter, planets are starting to cool down considerably.

The first two planets are hot and do not allow life on them.

The next two are most conducive to life, as we know it.

\section{Results}

In a few billions of years the sun will start to climb and it will expand a lot in its aging process, with the main halves ending up with a lot of Jupiter, Saturn, Uranus and Neptune. It will be almost impossible to live on Terra because of the extreme heat and probably the same on the small planet Mars.

Terra is the third planet from the Sun Fig. 9, a favorable position now for life (not too hot or too cold, sufficient light, average energy equivalent to life).

Our planet seen from space looks like the figure below. For now is the only known planet with real life conditions.

The earth is surrounded by a very strong magnetic field that sustains life on Earth Fig. 10. These lines of power make our planet a special one (maybe unique). They can't be seen with the naked eye because they are not located in the visible spectrum. In addition, Terra is surrounded by multiple spheres of electromagnetic waves, various frequencies, or various radiations, all of which are designed to protect us from the unfriendly radiation from the sun or from outer space. Both atmospheric and ozone shields play an essential role in our protection against gamma radiation, etc.

Terrestrial magnetic field is that actually supports life on Terra. You can see the lines of force; with blue symbolizes the north pole and the southern yellow.

Above the continents and oceans there is a thick coating called the atmosphere Fig. 11, whose existence is absolutely necessary for the unfolding of life on Earth. This dynamic coating is in constant interaction with the relief, the surface of the soil, the oceans and the ice, through permanent streams of energy and substance, providing the environment favorable to the emergence, development and maintenance of the biosphere. 


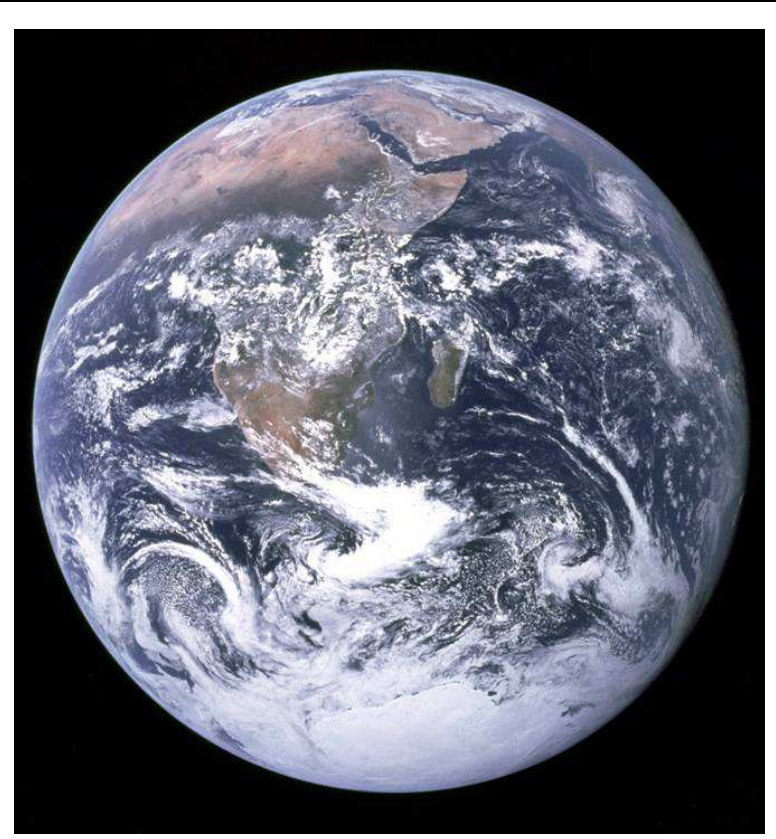

Fig. 9. Earth seen from space. Source: https://www.nasa.gov/multimedia/imagegallery/im age_feature_329.html

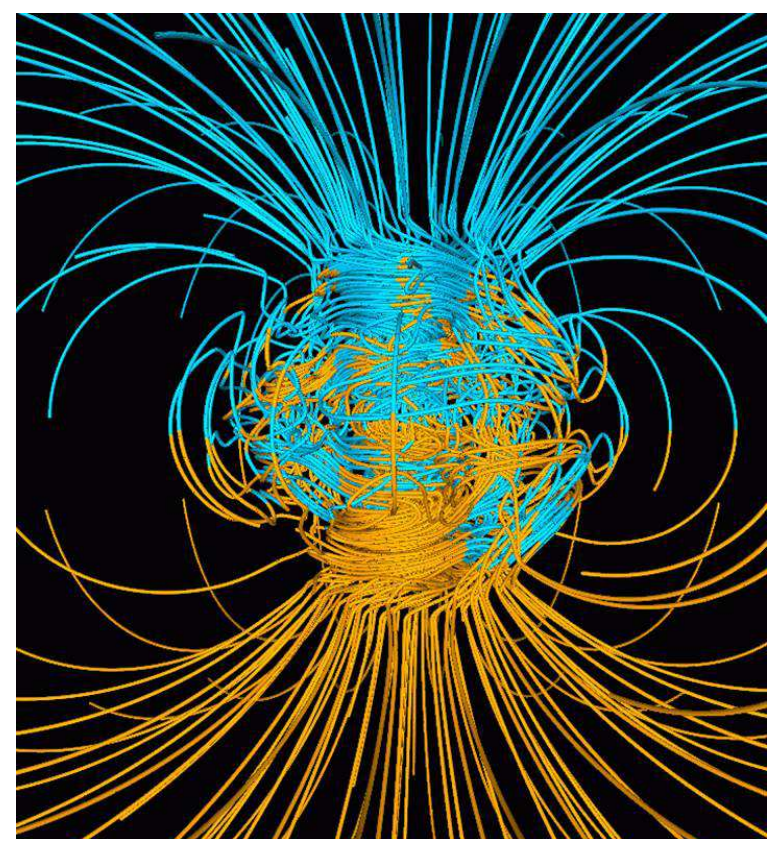

Fig. 10. Earth's magnetic field. Source: https://en.wikipedia.org/wiki/Earth\%27s_magnetic_field

The ozone layer is located at the top of the atmosphere (stratosphere) at the limit of space and acts as a shield, absorbing UVB (type B ultraviolet radiation) from the sun or cosmic space.

UVBs are very dangerous for people and vegetation, causing skin burns, cancer and destroying plant chlorophyll.

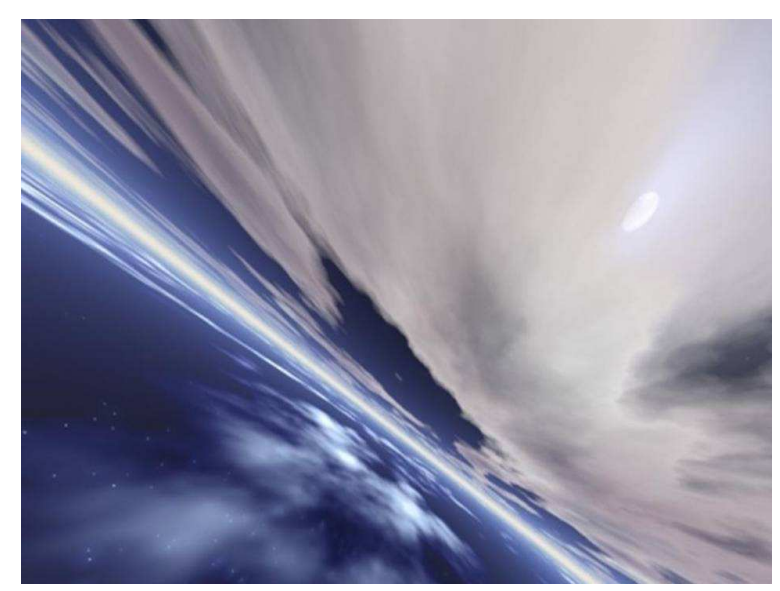

Fig. 11. The

atmosphere

Source: https://en.wikipedia.org/wiki/Atmosphere_of_Earth
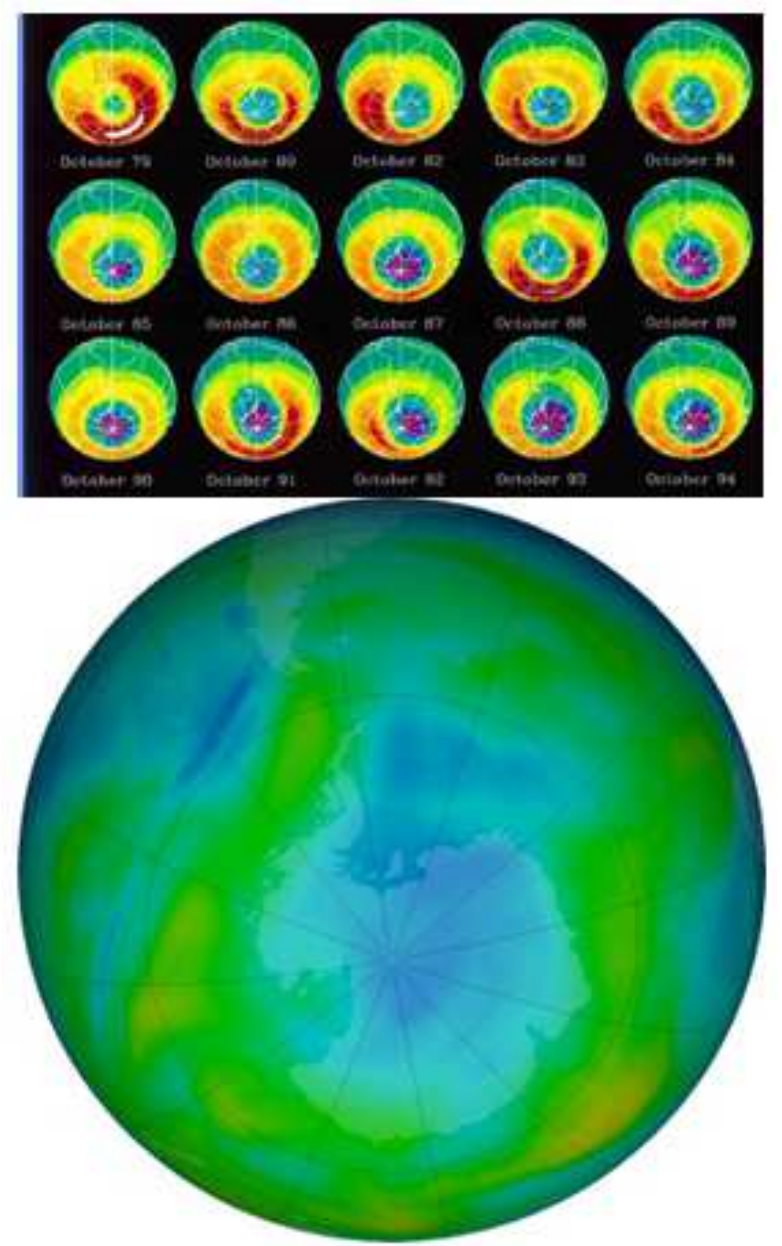

Fig. 12. Evolution of the Earth's ozone layer from 1979 to 1994 Source: https://ozonewatch.gsfc.nasa.gov/

The problem of thinning this layer finds a partial solution by replacing refrigerant in air conditioners and 
air conditioners as well as gas pressure from less harmful gas spray aerosols (sprays). Chlorofluorocarbon (CFC), which have been used for this purpose until recently (most commonly known as Freon), are largely responsible for destroying the ozone layer and have a pronounced greenhouse effect and their use is restricted or Banned in many countries.

Unfortunately CFC remains in the stratosphere for a long time (it has a lifetime of 50-100 years), slowing down the recovery of the ozone layer very much.

The ozone shield is constantly replenished by lightning through the earth's atmosphere. Approximately 300 lightning per second are produced on average on the stratosphere, strongly ionizing the stratospheric oxygen and thus permanently restoring the ozone layer surrounding Terra Fig. 12.

Much of ozone is destroyed to defend our planet. At one point, the amount produced was lower than the one consumed so that the ozone layer was thinned and drilled.

The rains were also fewer (so fewer lightning) due to the massive cutting of the terrestrial forests.

With the reforestation of the planet, rains began to multiply and with them, the lightning produced and thus the amount of ozone produced. The shield began to recover naturally and the hole in it gradually diminished (Petrescu et al., 2016a; 2016b).

\section{Discussion}

Without nuclear experiments (internationally forbidden because they were effectively disconnected from the Earth's ozone shield), no froes (banned worldwide), with a much more environmentally-friendly refurbished industry, the one still destroying us from the ozone shield is "the friend our car ".

Unfortunately, in less developed countries, massive forest cuts have begun and the planet is again put in danger.

The process of forest protection and reforestation must continue to be organized through government programs. Every planted but well-groomed tree saves the balance of our "mother planet."

Forests always generate the ozone layer directly and indirectly by generating clouds and then rains and lightning.

Forests also give the oxygen so vital to life and extend the carbon in the air and its various compounds, extremely toxic to humans and animals in general.

These compounds have arisen in particular from the combustion of large enterprises, from fossil fuel power plants (coal, wood, oil, gas) and especially from road vehicles, in the case of cars with internal combustion engines In particular), the planetary fleet currently reaching a huge level of about 1 billion cars in circulation simultaneously.

With all the measures taken to limit the emissions of pollutants, there are more and more and no wonder, given that the "car king" has multiplied from a few copies in 1866 to about one billion vehicles today, while new tens and tens of millions of vehicles are produced With all the measures taken to limit the emissions of pollutants, there are more and more and no wonder, given that the "car king" has multiplied from a few copies in 1866 to about one billion vehicles today, while new tens and tens of millions of vehicles are produced annually. The car king who has already carried us 150 years, destroys our health and the planet every day (Aversa et al., 2016a; 2016b; 2016c; 2016d; 2016e; 2016f; Mirsayar et al., 2017).

Approximately 300 beats per second cross the terrestrial atmosphere on average Fig. 13. Flash is an example of plasma present at the Earth's surface. It represents an electrical discharge at a voltage exceeding 100 million volts, generating an electrical current of more than 30000 amperes, emitting light, radio waves, X-rays, gamma radiation, a powerful noise (thunder) and producing a strong ionization of the earth's atmosphere Oxygen in ions and the production of ozone in large quantities.

There are also two other terrestrial shields, one X-ray and one gamma radiation. All four shields are designed to protect our planet from the destructive radiation coming from outer space (in general).

Light bulbs create power and keep the ozone shield from the stratosphere. It also generates a larger radio shield around the Earth at high heights. There are also two other terrestrial shields, one X-ray and one gamma radiation (Petrescu and Calautit, 2016a; 2016b). All these four shields have the role of defending our planet of destructive radiation coming from outer space (in general).

Thousands of years ago, the atmosphere of our planet contained $30 \%$ oxygen. Today, due to massive deforestation and carbon dioxide pollution, the amount of oxygen in the Earth's atmosphere has fallen to $21 \%$.

In large cities, pollution is much higher, so the amount of oxygen can drop to $18 \%$. The human body works optimally at $30 \%$ oxygen, but has adapted to $21 \%$; if this percentage drops to $18-21 \%$ the body is negatively affected. Nowadays, human blood carries a much smaller amount of oxygen, the tissues leading to a permanent lack of oxygen and therefore the immune system is much weaker, like the circulatory, respiratory and especially the central nervous system (people tend to Resist much less stress factors and nerve diseases, along with respiratory diseases).

The low amount of oxigen in the earth's atmosphere, the much diminished percentage (over time atmospheric oxygen has halved, over the past 300 years has continued to decline by a few more percentages) is actually the main problem of our planet, along with global warming.

Both major problems are caused by massive 
deforestation and the global industrialization process by which the carbon dioxide in the atmosphere is depleted to the detriment of oxygen.

"More and more polluting and highly polluting technologies have done over the past 300 years that our planet is not as blue as it was at first!"

Pollution began with the industrialization of the planet 300 years ago. The highest and lasting pollution is due to the heavy industry and the car, but the explosions of atomic and nuclear experiments also played a role in the destruction of ozone Fig. 14.

Although not officially announced, it is known that the massive destruction of the ozone shield with its drilling, but also the major imbalances of the planet, is due to nuclear weapons tests.

They also produced mutations, malformations and genetic anomalies, multiplying diseases including incurable ones.

They poisoned soil, water, air and long-term organisms.

Modern, electric, hydrogen, or mixed (hybrid) cars will solve the problem of pollution with carbon monoxide and carbon dioxide. Combustion hydrogen produces only water that it gives to its natural circuit Fig. 15.

Almost all of the globe is giving up coal fuels (extremely pollutants). Slowly, but surely, oil, gas, hydrogen, nuclear power, or directly from the Sun are being switched on by the capture and conversion of light into electricity using photovoltaic cell panels Fig. 16.

Much stronger and more cost-effective are the solar farms built on a larger surface, which have a central tower and around it as more parabolic mirrors all focus on the central tower Fig. 17.

The mirrors reflect the concentrated luminous energy received from the sun focusing it all on an area of the tower where there is a boiler with water or other liquid (agent) that is overheated and which will operate a steam turbine that will move an electric generator (or A stirring-generator group). The installed power of these solar farms is very high.

A very good yield is also provided by wind farms Fig. 18, which can be installed almost anywhere, provided that the area is constantly beating.

As the earth is stronger and faster and the power produced will be higher.

Modern wind turbines produce a power of between $600 \mathrm{~kW}$ and $5 \mathrm{MW}$, the most used being the 1.5-3 MW output power, making them simpler and more suitable for commercial use. Nowadays, even today, 10-30 times more powerful wind turbines have been invented and built, with a much higher energy efficiency, being extremely efficient in mounting them in suitable places, as only 10 such devices can generate The energy given by a fission power plant (a reactor).

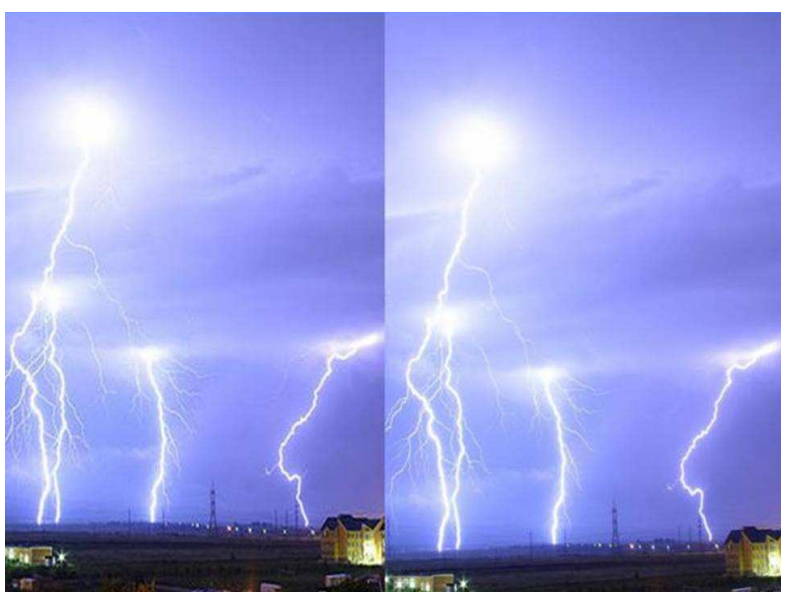

Fig. 13. Approximately 300 beats per second cross the terrestrial atmosphere on average Source: https://en.wikipedia.org/wiki/Lightning

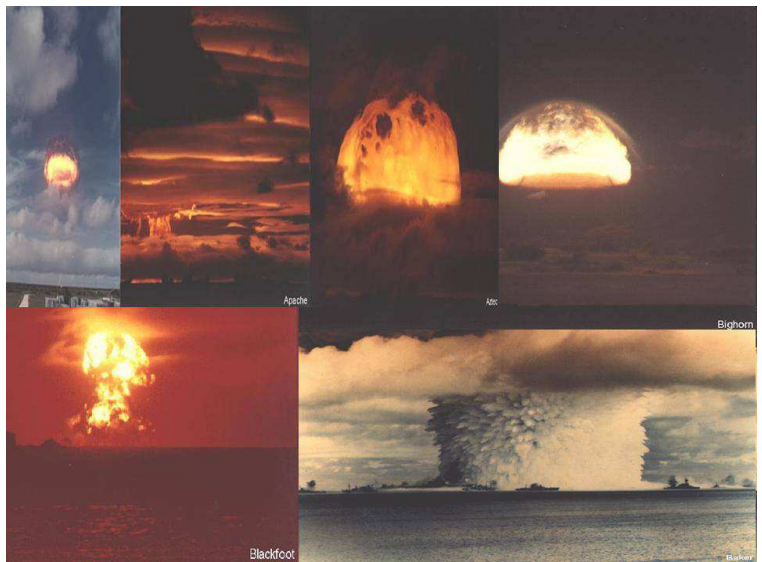

Fig. 14. A major role in the destruction of ozone was also played by the explosions of atomic and nuclear ground experiments; have poisoned soil, water, air and longterm organisms Source: https://en.wikipedia.org/wiki/Nuclear_weapons_testing

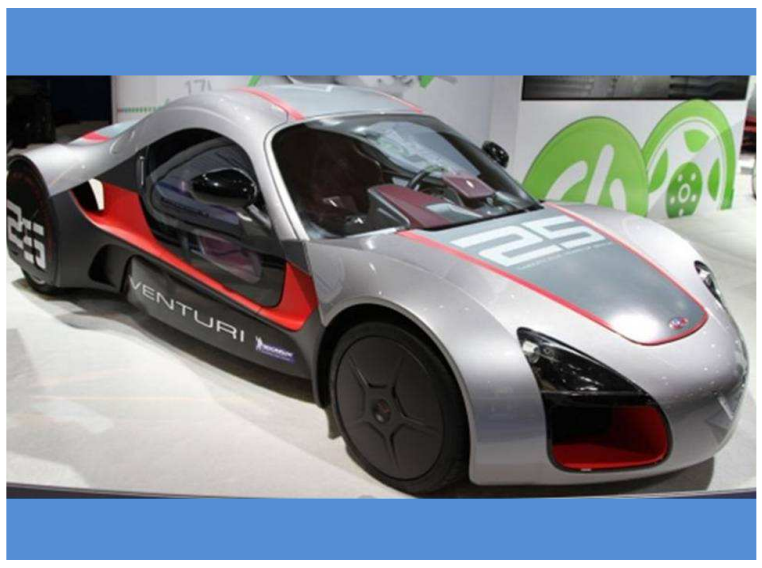

Fig. 15. Modern, electric cars 


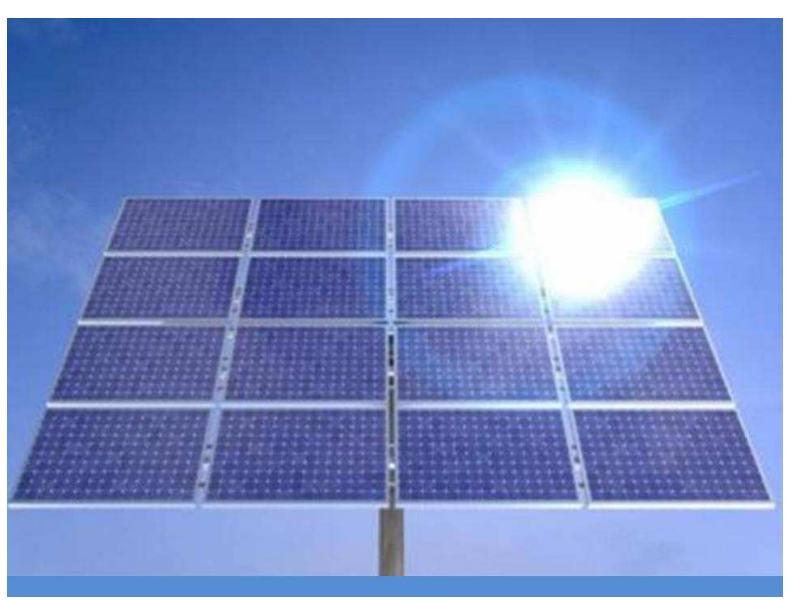

Fig. 16. Green, renewable, sustainable, clean, friendly energy, obtained with solar photovoltaic panels

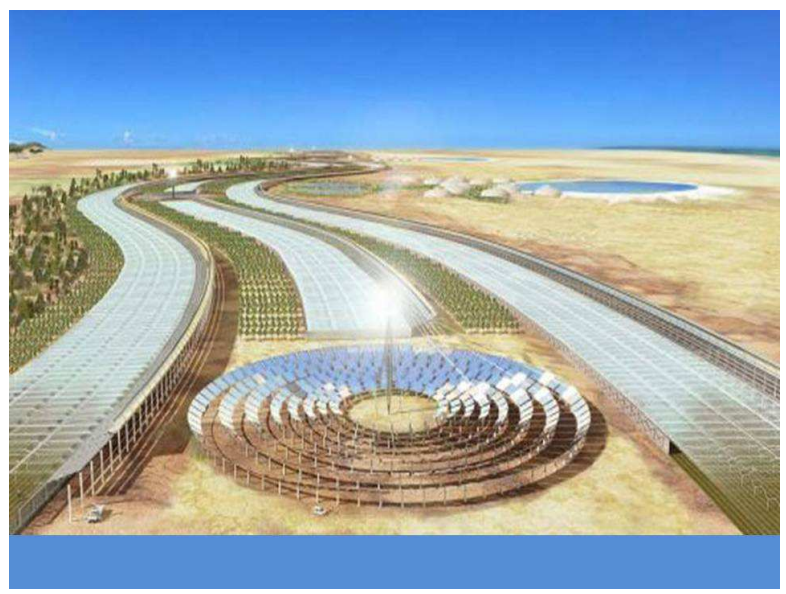

Fig. 17. Green, renewable, sustainable, clean, friendly energy, obtained with the help of a solar farm in the center of the image; and in the sides are photovoltaic panels

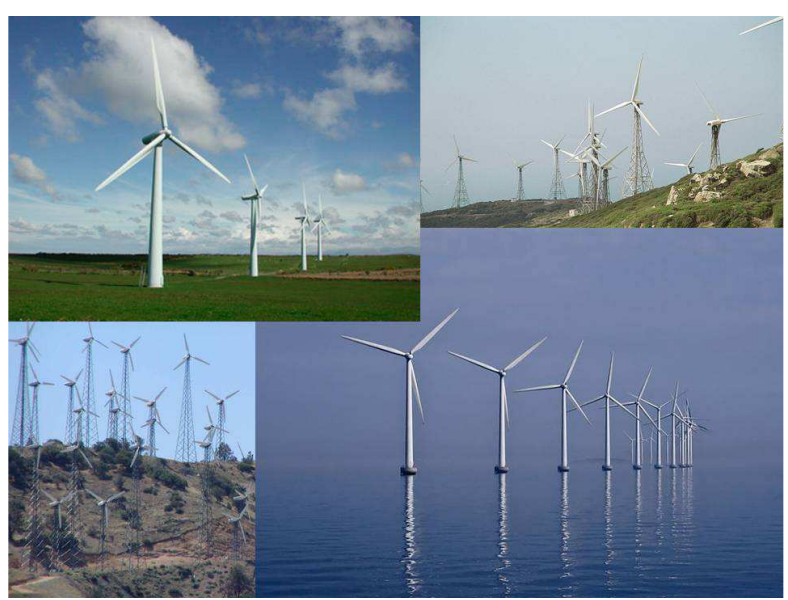

Fig. 18. A very good yield is also provided by wind farms, which can be installed almost anywhere, provided that the area is constantly beating
Only 100 of these arrays can generate the energy of about 10 nuclear reactors (representing an installed power of about $6 \mathrm{Gw}$ ), which is the energy needs of a highly developed and industrialized country.

Biomass Fig. 19 can't be eliminated immediately, as it would be desirable, for the simple reason that it still represents a very high energy percentage and for the moment, humanity is in full swing and expansion is constantly increasing its energy consumption without New emerging energy sources bring significant percentages, as oil reserves are endangered.

Though not mentioned and not officially communicated, since 1970 our planet has entered into an energetic semicripsis, with syncope, with increases and decreases, solved locally, partially but not definitively. The highest percentage of energy growth since then has been achieved by nuclear fission energy (19-20\%) and biomass (around 14\%).

Nuclear power and biomass have thus managed to take together about $33-34 \%$ of the world's energy consumption. Both are sustainable, independent energy sources (biomass being totally renewable).

Fortunately, today wind and solar have been developed and implemented in a very large amount, increasing over the last three to four years from $1 \%$ to about $10-15 \%$.

Classical energies represent for the moment but also for the immediate future a convenient, safe and handy reserve. Biofuels will be used more and more, as we have done since ancient times, as long as we fail to find a sufficient alternative, cheap, convenient, direct, renewable, nonhazardous, non-polluting alternative energy.

The gas, continues to be a strategic natural reserve of the planet. Whether they are associated with oil reserves or are in separate fields, they have played an essential role in the last 150 years and will be equally important in the future. If at first they were treated with contempt, using only the oil, being burned or simply scattered into the atmosphere, today the gases are used both for industrial purposes and for domestic needs. We should burn them only for cooking and heat to make them electric or otherwise to consume them in smaller quantities and to protect and preserve them for a longer time, we all think most of the time; Yes, but if the electricity comes not from the new energy technologies (solar, wind, etc.), or from hydropower plants or nuclear power plants, then the electricity consumed to protect the natural gas reserves comes mostly from the gas Burned (or burned oil) in power plants. In this case, there will be no gas savings, but an increase in actual consumption of natural gas burned (thanks to conversion losses).

The same thing happens when we remove a classic gasoline, gasoline or gas engine and pass it on to hydrogen or replace it with an electric one.

The vehicle consumes electricity from some modern batteries, which are charging from sockets (more modern directly through electromagnetic waves, or other system without sockets and connections). 


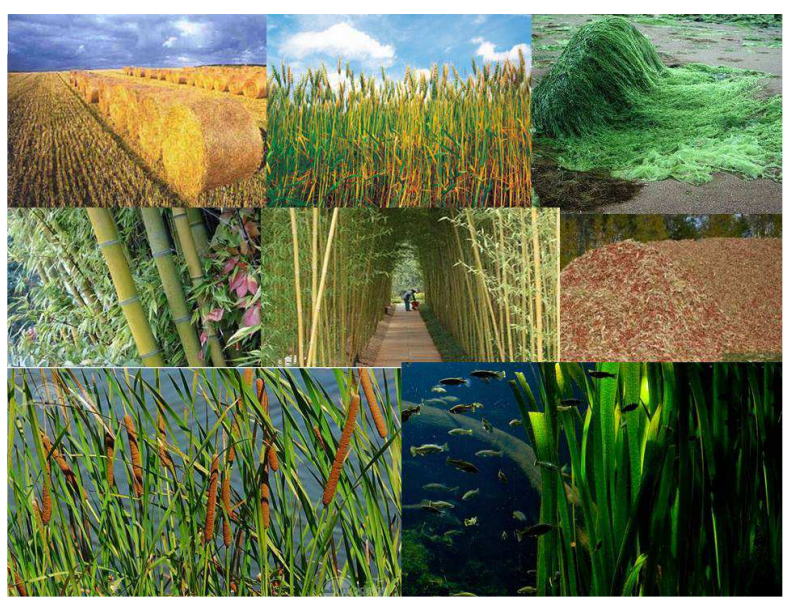

Fig. 19. Biomass is totally renewable

The current is produced in the proportion of $66 \%$ of the gas and/or oil burning in the thermal power stations and the required electric current will produce an additional consumption of natural gas anyway higher than the oil or gas produced by the classic engine from the vehicle.

Finally, instead of a gas economy, we produced an extra hole in the planet's strategic gas reserves.

Let's assume that instead of electric motors we choose a hydrogen engine to take the place of a classic one on hydrocarbons (oil, gas). The equivalent energy (most of all produced from the flue gas) consumed to produce hydrogen is higher than the energy given by the hydrogen thermal engine, so we already have a real unfavorable gas yield from the start, which will be consumed additionally by the replacement. But things do not just stop here; In the case of hydrogen, it must be liquefied and bottled and the equivalent energy required for this additional operation is now about ten times higher than that obtained by burning hydrogen in the adapted heat engine.

In other words (more plastic) by replacing a classic hydrocarbon engine with an electric one, the equivalent (real) gas (and/or petrol) consumption increases about 1.3 times instead of falling (planetary) and if the classic engine Will be replaced with one on hydrogen then the consumption of gas (or flue gas) will increase about 11.3 times.

In order to be able to introduce as many electric motors as possible in real terms and with a decrease in the actual use of oil on a planetary scale, it is necessary to reduce the percentage of natural gas and oil used for heating and electricity generation by increasing the number of power plants Nuclear, wind, solar, hydro, etc.

The procedures are not as easy as it would appear at first sight, because when we proudly announce that the number of wind and solar power plants has increased by about $30 \%$, this increase is related to the existing ones and even without considering them Worn out, an increase of $30 \%$ of about $2-3 \%$ of new existing renewable means a real annual absolute increase of the planetary weight of new regenerable energies from 2-3 to $2.7-4 \%$, i.e., an increase in Real terms of new energies of $0.7-1 \%$, which would mean very little in the conditions of maintaining constant planetary consumption. If planetary consumption would be constant with an annual increase of about $0.7 \%$, new energies could replace those obtained from the burning of hydrocarbons in about 95 years and by then they could be depleted long before, the planet and mankind Thus entering into an extremely serious crisis that would not only be energetic.

The problem of survival, the return to caves, of wars for the extermination of the human race would be effectively addressed, which is not yet on the ground, but it is continuously multiplying by requiring more and more planetary resources including energy.

The problem is far more serious than at first glance, because the energy consumption of the planet does not stay, but it grows by about $1-3 \%$ annually.

An increase in the planet's annual energy consumption of only $0.7-1 \%$ automatically cancels the growth of new renewable energies and the additional increase in energy consumption means that the new renewable energy actually drops annually in planetary proportions, from $4-5$ to $2-3 \%$ and probably even below in the future, to the astonishment of those who were expecting to see them actually growing because there are more and more.

The obvious solution is that new renewables will grow yearly at an even faster rate, at least by their annual doubling, i.e., reported at their level to suffer an annual increase of not $30 \%$ but at least $100 \%$.

So we can put the planet on a normal path, starting from new renewable, non-polluting energies.

Separately, we will continue to use biofuels more and more, but also new nuclear power plants alongside existing ones.

We should also increase the hydro power plants wherever possible.

Any new energy source is welcome!

The discovery of new deposits of natural gas and oil is also announced.

All must be seriously considered, rationalized, consumed immediately, or strategically preserved to be consumed later. No uncovered reserve should be abandoned or disregarded. At least for the moment we can't afford to disregard the classical energy reserves.

"What's in your hand is not a lie!"

The gas industry has gone into a new phase, the exploitation of unconventional resources. They have transformed the US into the world's largest gas producer. Estimates of the institutions show that mankind's gas reserves are actually more than $40 \%$ higher than previously known, due to unconventional resources. 
It is not in vain that natural gas is called "blue gold." Like oil, where the synonym of "black gold" no longer surprised anyone, the gases became vital to human civilization. In the past, the great explorations aimed at oil discoveries and often when only gases were found, the disappointment was profound and the gases were simply burned into the atmosphere without any purpose. Today, billions of Euros are spent on explorations and a dozen times more for gas extraction.

But the industry has evolved so quickly that traditional gas has been overcome and resources already declared as unconventional are already extracted Fig. 20.

Unconventional gases are actually natural gas, but they are extracted from hard rocks and hard to explore. Therefore, unlike classical vertical probes, the new resource category needs another technology.

USA, the world leader in unconventional (shale)

In recent years, Americans have made a significant advance in this area and have developed equipment that seems to be in the SF field.

Controversially, shale gas has already been able to make a significant (energy) contribution at planetary level.

Practically, the probes, vertically penetrating the ground, are horizontally inserted into deep rocks of hard rock.

There are cracks where the gases are gathered, which are then collected and brought to the surface.

The state-of-the-art technology allows extraction from bituminous shale, clay, sandy rocks and coal beds.

There is more talk about shale gas, as these new ultra-strategic reserves are called (because they are also extracted from bituminous shale).

Anyway, they are coming to prolong the life of traditional energy reserves. It is an oxygen bubble for humanity, because in the time span we can try and develop new energy technologies.

In 2003, the US National Petroleum Council estimated that North America could have reserves of 1.1 trillion cubic meters of shale gas. This year, the US Advanced Resources International Institute showed that there could be 50 times more resources there.

In April, the US Energy Information Administration issued a report that, since 2000 , the development of the unconventional resource sector has revealed that mankind's gas reserves are actually more than 40\% higher than previously known.

Already, Canada has discovered shale gas, that is, unconventional gas, in Apalai and British Columbia.

In Poland, it appears to be the largest shale gas field in Europe, estimated at 5,300 billion cubic meters.

And in Ukraine shale gas reserves are apparently up to at least 30 trillion cubic meters (a huge planetary energy reserve).

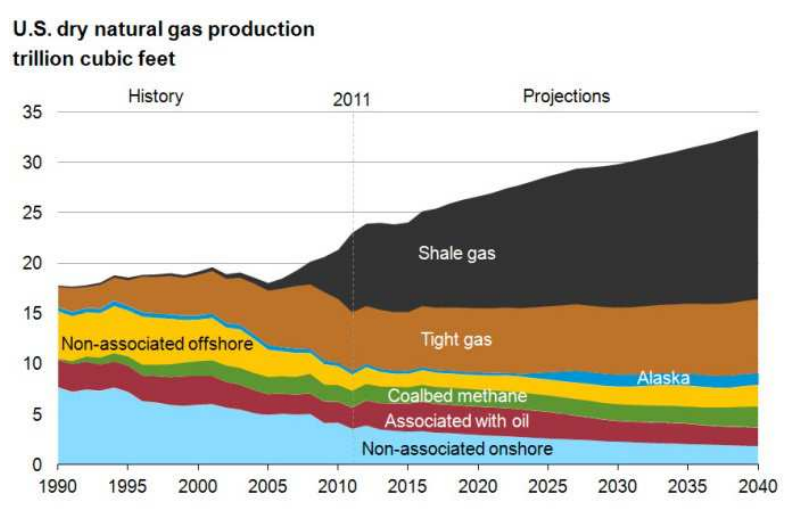

Source: U. S. Energy Information Administration, Annual Energy Outlook 2013 Early Release

Fig. 20. Exploitation of shale gas Source: https://en.wikipedia.org/wiki/Shale_gas_in_the_Unit ed_States\#/media/File:US_Natural_Gas_Production_ 1990-2040.jpg

\section{Conclusion}

If thinning of the ozone layer is responsible for part of the global warming phenomenon, leaving more sunlight to reach the Earth, increasing the amount of nitrogen oxide, methane, carbon monoxide and carbon dioxide in the atmosphere amplifies this phenomenon by accelerating the effect of greenhouse, what today is called "global warming," representing the number one problem of the planet.

Due to global warming, the climate of the planet is changing. Everywhere in temperate areas the appearance of seasons changes; Winter is stretching; Summer is warmer; Temperatures have greater and more sudden variations.

It melts from the North and South poles, leaving many penguins and white polar bears mortal.

The water level increases and the dryness decreases. The climate is deteriorating; so do the terrestrial atmosphere, the waters and the oceans. The storms are amplified and multiplied.

The summer heat is sometimes unbearable (instead of the maximum of $25-28^{\circ} \mathrm{C}$ we had even the maximum of $44^{\circ} \mathrm{C}$ ).

Animals innocent and helpless as if shouting out of their last powers: "Good people do something with the Blue Planet! ..."

The polar bears isolated on an ice bank (you are destined to starve before all the ice melts and they drown) "as if they are goodbye to us!" Also, the penguins, disoriented, are floating on a piece of ice until it is exhausted by hunger and fatigue, or until all the ice melts.

Although not officially announced, it is known that the massive destruction of the ozone shield with its drilling, but also the major imbalances of the planet, is due to nuclear weapons tests. 
They also produced mutations, malformations and genetic anomalies, multiplying diseases including incurable ones.

They poisoned soil, water, air and long-term organisms.

The blue planet is unique in the universe, by its mode of compilation.

It is clear to everyone that such an inheritance must be preserved so that it can also be used by our descendants.

Man was not created just to eat, have fun and perpetuate the species like animals.

Having in him the mysterious light of divine creation, man is destined to evolve permanently, now being the time when he will become a conqueror of the universe.

Strong telescopes are needed to thoroughly study the entire universe and to discover new living planets.

We need modern spacecraft, new technologies, weapons and robots to explore and conquer the universe we are in.

We need new forms of energy, clean, friendly, sustainable, renewable, concentrated.

The first necessary step is the commencement of the construction of bases on Mars, with the obvious aim of launching a Martian based terrain project.

\section{Acknowledgement}

We acknowledge and thank $\mathrm{Mr}$ Taher M. AbuLebdeh, Associate Prof at North Carolina A and T State University, United States, Mr Muftah H. El-Naas PhD MCIC FICCE QAFCO Chair Professor in Chemical Process Engineering Gas Processing Center College of Engineering Qatar University and Ms Shweta Agarwala, Senior Research Scientist at Singapore Center for 3D Printing Nanyang Technological University Singapore for their suggestions and comments.

\section{Funding Information}

Copyright: 819-kbHquxwykr.

\section{Author's Contributions}

All the authors contributed equally to prepare, develop and carry out this manuscript.

\section{Ethics}

This article is original and contains unpublished material. The corresponding author confirms that all of the other authors have read and approved the manuscript and no ethical issues involved.

\section{References}

Aversa, R., D. Parcesepe, R.V. Petrescu, G. Chen and F.I.T. Petrescu et al., 2016a. Glassy amorphous metal injection molded induced morphological defects. Am. J. Applied Sci., 13: 1476-1482.
Aversa, R., R.V. Petrescu, F.I.T. Petrescu and A. Apicella, 2016b. Smart-factory: Optimization and process control of composite centrifuged pipes. Am. J. Applied Sci., 13: 1330-1341.

Aversa, R., F. Tamburrino, R.V. Petrescu, F.I.T. Petrescu and M. Artur et al., 2016c. Biomechanically inspired shape memory effect machines driven by muscle like acting NiTi alloys. Am. J. Applied Sci., 13: 1264-1271.

Aversa, R., R.V. Petrescu, R. Sorrentino, F.I.T. Petrescu and A. Apicella, 2016d. Hybrid ceramo-polymeric nanocomposite for biomimetic scaffolds design and preparation. Am. J. Eng. Applied Sci., 9: 1096-1105.

Aversa, R., V. Perrotta, R.V. Petrescu, C. Misiano and F.I.T. Petrescu et al., 2016e. From structural colors to super-hydrophobicity and achromatic transparent protective coatings: Ion plating plasma assisted $\mathrm{TiO}_{2}$ and $\mathrm{SiO}_{2}$ Nano-film deposition. Am. J. Eng. Applied Sci., 9: 1037-1045.

Aversa, R., R.V. Petrescu, F.I.T. Petrescu and A. Apicella, 2016f. Biomimetic and evolutionary design driven innovation in sustainable products development. Am. J. Eng. Applied Sci., 9: 1027-1036.

Charles Messier, from Wikipedia. https://en.wikipedia.org/wiki/Charles_Messier

Mirsayar, M.M., V.A. Joneidi, R.V.V. Petrescu, F.I.T. Petrescu and F. Berto, 2017. Extended MTSN criterion for fracture analysis of soda lime glass. Eng. Fracture Mechan., 178: 50-59. DOI: 10.1016/j.engfracmech.2017.04.018

Atkinson, N., 2015. Do "skeleton" filaments give structure to the universe? Univers today.

Petrescu, F.I. and J.K. Calautit, 2016a. About nano fusion and dynamic fusion. Am. J. Applied Sci., 13: 261-266. DOI: 10.3844/ajassp.2016.261.266

Petrescu, F.I. and J.K. Calautit, 2016b. About the light dimensions. Am. J. Applied Sci., 13: 321-325. DOI: 10.3844/ajassp.2016.321.325

Petrescu, RV., R. Aversa, A. Apicella, S. Li and G. Chen et al., 2016a. Something about electron dimension. Am. J. Applied Sci., 13: 1272-1276.

Petrescu, F.I., A. Apicella, R.V. Petrescu, S. Kozaitis and R. Bucinell et al., 2016b. Environmental protection through nuclear energy. Am. J. Applied Sci., 13: 941-946.

DOI: 10.3844 /ajassp.2016.941.946

Virgo Constellation From Wikipedia, the free encyclopedia.

https:/en.wikipedia.org/wiki/Virgo_(constellation) 